\title{
MEETINGS
}

\section{Congress of ANesthesiology}

Sponsored by the Hungarian Society for Anaesthesiology and Reanimation September 2-4, 1975

For information :

Belatonfured, Hungary

Medical Societies (Motesz)

H 1361 Budapest, POB 32

Hungary

Mr. Gio Fischer

2 Carlton Street

Toronto, Ontario, Canada

The Tenth Annual Educational Meeting of the

Canadian Society of Respiratory Technolocists

will be held

September 3, 4, 5, 1975,

For information:

at Halifax, Nova Scotia

Mrs. T. Cashen, R.R.T.

c/o Respiratory Technology Department,

Victoria General Hospital

Halifax, Nova Scotia

Congress of the Belgian Society of Anesthesia and Reanimation

Brussels, Belgium

September 11-14, 1975

For information:

Prof. Dr. G. Rolly

Dienst voor Anesthesie

Akademisch Ziekenhuis

De Pintelaan 135

B-9000 Ghent (Belgium)

Obstetric Anaesthetists' Association

(UNITED KingDom)

University of Bradford

September 12,1975

University of Nottingham

March 26, 1976

For information:

Dr. B.A. Waldron, Honorary Secretary

Obstetric Anaesthetists' Association

Nottingham City Hospital

Hucknall Road

Nottingham NG5 1PB

United Kingdom 
Ontario Division Canadian Anaesthetists' Society AND

Anaesthetic Section

Ontario Medical Association Fall Meeting October 2-3, 1975

Waterloo Motor Inn, Waterloo For information:

Dr. G.W. Kinsman 900 King Street West Kitchener, Ontario N2G 1G5 Canada

The Hospital for Sick Chrodnen (1876-1975)

Centennial Edition

OF OUP

Paediatric Anaesthetic Conference

November 7-9, 1975

For information:

Dr. D.J. Stewart

Paediatric Anaesthetic Conference

The Hospital for Sick Children

555 University Avenue

Toronto, Ontario

M5G 1X8

\title{
INTERNational ANESTHESIA SOCIETY 50th Congress \\ Caesar's Palace, Las Vegas, Nevada March 14-18, 1976
}

For Information:

Dr. B.B. Sankey

Executive Secretary

Intemational Anesthesia Research Society

3645 Warrensville Center Road

Cleveland, Ohio 44122 U.S.A.

Sixth World Congress of Anesthesiologists

$$
\text { Mexico City }
$$

For information:

$$
\text { April 24-30, } 1976
$$

\author{
Dr. Enrique Hulsz \\ General Secretary \\ Amsterdam No. 14-303 \\ Mexico 11, D.F., Mexico
}


Canadian Anaesthetists' Society Annual Meeting

June 20-24, 1976

Queen Elizabeth Hotel, Montreal, Quebec

For information:

The Honorary Secretary

Canadian Anaesthetists' Society

178 St. George Street

Toronto, Ontario

M5R 2M7 Canada 